\title{
Identification of multiple pathways involved in the malignant transformation of endometriosis (Review)
}

\author{
YUMI HIGASHIURA, HIROTAKA KAJIHARA, HIROSHI SHIGETOMI and HIROSHI KOBAYASHI \\ Department of Obstetrics and Gynecology, Nara Medical University, Nara, Japan
}

Received November 26, 2012; Accepted April 18, 2012

DOI: $10.3892 / \mathrm{ol} .2012 .690$

\begin{abstract}
The association between endometriosis and malignant transformation has often been described in the medical literature. A search was conducted between 1966 and 2010 through the English language literature (online Medline PubMed database) using the keywords endometriosis combined with malignant transformation. The search revealed an increase in reports describing endometriosis and malignancy. Approximately $1.0 \%$ of women with endometriosis have lesions that undergo malignant transformation. The malignant processes that are associated with endometriosis may be classified into three groups: i) epithelial ovarian cancers (endometrioid adenocarcinoma and clear cell carcinoma), ii) other Müllerian-type tumors, including Müllerian-type mucinous borderline tumor and serous borderline tumor and iii) sarcomas such as adenosarcoma and endometrial stromal sarcoma in the female pelvic cavity. Persistent oxidative stress induced by endometriosis-dependent hemorrhage may be associated with carcinogenesis. In conclusion, the malignant transformation of endometriosis has multiple pathways of development and may share a common pathogenic mechanism; iron-induced oxidative stress derived from repeated hemorrhage.
\end{abstract}

\section{Contents}

1. Introduction

2. Review of the literature

3. Endometriosis-associated malignant transformation

4. Endometrioid adenocarcinoma of the ovary

5. Clear cell carcinoma of the ovary

6. Serous borderline tumor

Correspondence to: Dr Hiroshi Kobayashi, Department of Obstetrics and Gynecology, Nara Medical University, 840 Shijo-cho, Kashihara, Nara 634-8522, Japan

E-mail: hirokoba@naramed-u.ac.jp

Key words: endometriosis, ovarian cancer, serous borderline tumor, Müllerian mucinous borderline tumor, adenosarcoma, endometrial stromal sarcoma
7. Müllerian mucinous borderline tumor

8. Adenosarcoma

9. Endometrial stromal sarcoma

10. Discussion

\section{Introduction}

Endometriosis is a common gynecological condition affecting $10 \%$ of women of childbearing age. It is a chronic disease that is characterized by ectopic endometrial tissue outside the uterus, typically leading to painful symptoms, dysmenorrhea, dyspareunia, pelvic pain, infertility, and malignant transformation (1). It has been reported that endometriosis can be a precursor of some ovarian cancers. Previous studies have shown that endometriosis has the potential to induce several types of premalignant lesions (1-3).

Herein, we reviewed the malignant transformation of endometriosis and described the mechanisms whereby genetic and microenvironmental factors contribute to the neoplastic progression of endometriosis.

\section{Review of the literature}

A comprehensive review of the literature was conducted to investigate record numbers of 'malignant transformation of endometriosis' reported worldwide. A Medline search of the literature was performed using the keywords endometriosis, cancer, tumour, tumor, carcinoma, adenocarcinoma, sarcoma, malignancy, neoplasm, ovary, ovarian and extragonadal. English-language publications in PubMed and references from relevant articles published between January 1, 1966 and December 31, 2010 were analyzed. References in the studies identified were also searched, and some unpublished data were obtained.

Between 1966 and 2011, the literature search identified 483 reports, with the incidence of reports increasing over time (Fig. 1). Of these 483 reports, 432 (89\%) described epithelial malignancy and only $51(11 \%)$ described mesenchymal malignancy. Data describing endometriosis and epithelial malignancy from the PubMed database, encompassing the years 2001 to 2010 , clearly show a marked increase of reports. The number of reports describing endometriosis and mesenchymal malignancy has also increased, but less rapidly than those describing endometriosis and epithelial malignancy. 


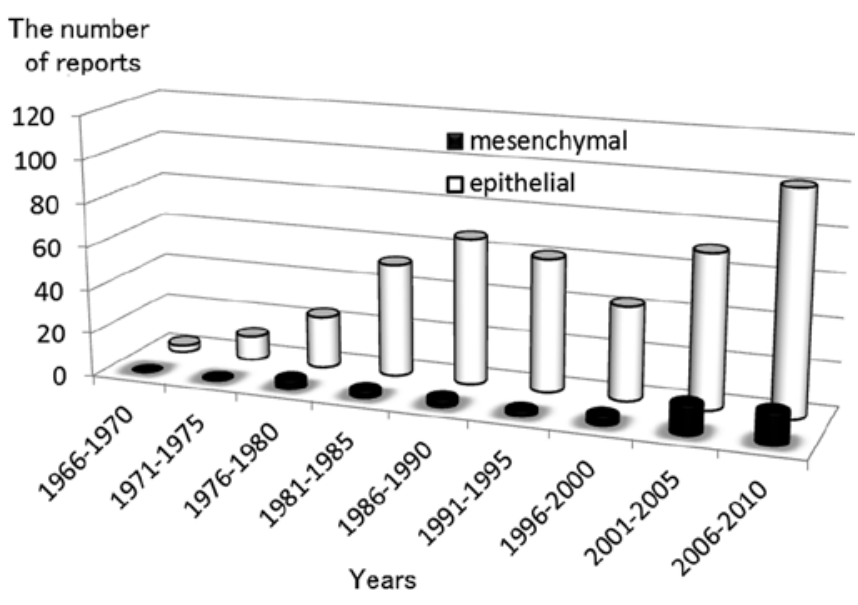

Figure 1. Trends in the number of reports describing endometriosis and malignancy from the PubMed database, encompassing the years 1966 to 2010. The gray columns show the number of reports describing endometriosis and epithelial malignancy. The black columns show the number of reports describing endometriosis and mesenchymal malignancy. The number of reports continued to increase for both malignancies.

\section{Endometriosis-associated malignant transformation}

Endometriosis is a complex disease with a multifactorial pathogenesis. Extensive investigation has explored the role of genetics, epigenetics, environmental factors and ethnicity in predisposing women to develop endometriosis (1-3). Some studies reported a higher prevalence of upper respiratory infection, vaginal infection and pelvic inflammatory disease compared to the general population (2). Numerous immune factors are modified, not only within endometriotic lesions, but also within the eutopic endometrium (3). Thus, endometriosis is an inflammatory condition, associated with a dysregulated immune response (2).

Further studies reported a higher prevalence of melanoma, non-Hodgkin's lymphoma, ovarian cancer, endometrial cancer and breast cancer in women with endometriosis (2,4-6). Although endometriosis cannot be termed a premalignant disease, epidemiological, histopathological and molecular data suggest that this condition has malignant potential (7). The presence of endometriosis is associated with an increased risk of synchronous neoplasms in the ovary and endometrium, so-called endometrial and ovarian cancer synchronous to endometriosis (8). Concurrent endometrial pathology has been observed in cases of malignant transformation of endometriosis (approximately 30\% of cases) (9). In their study, Mabrouk et al reported a case of mixed clear cell and endometrioid type adenocarcinoma of the extragonadal, rectovaginal septum, arising from endometriosis (deep infiltrating endometriosis) and associated with a differentiated, endometrioid endometrial carcinoma (10). Since endometriosis is frequently found in association with malignancies, it could be viewed as a neoplastic process. Endometriosis might also be the product of numerous predisposing factors such as genetic abnormalities and genomic imbalances in specific chromosomes for the development of neoplasms (11). Accumulating evidence suggests a role for genetic activation and inactivation mutations. For example, ovarian cancers and adjacent endometriotic lesions have shown common genetic alterations, suggesting a possible malignant genetic transition spectrum $(12,13)$.

The exact incidence of malignant transformation of endometriosis is difficult to ascertain, since a strict criterion is the demonstration of a histologically proven transition from benign precursors to malignant lesions (14). Nonetheless, malignant transformation is not a rare complication of endometriosis and is estimated to occur in $0.6-0.8 \%$ of women with ovarian endometriosis $(15,16)$. The frequency of malignancy in surgically proven endometriosis is $>10 \%$ (9).

Cases of surgically proven endometriosis were reviewed to evaluate the associated types of pelvic cancers. Adenocarcinoma is the most common $(70 \%)$, and sarcoma is the second most common malignancy (12\%) (17). The ovary was the primary site in $79 \%$ of the cases with a malignant tumor arising in endometriosis, whereas extragonadal sites represented $21 \%$ of the cases (17). In the ovary, endometrioid adenocarcinomas accounted for $69 \%$ of disease, clear cell carcinomas $14 \%$, sarcomas $12 \%$, and rare cell types $6 \%$ (17). By contrast, clear cell carcinoma and adenosarcoma were most commonly observed in extraovarian endometriosis (9). A specific link between endometriosis and endometrioid and/or clear cell carcinoma of the ovary has been reported, with an odds ratio ranging between 3.7 and 35.4 (confidence interval 95\%) (18). Kobayashi et al evaluated the risk of ovarian cancer based on varying time periods from the time of diagnosis of ovarian endometriosis (19). During a follow-up period of up to 17 years, 46 incidental ovarian cancers were identified, translating into a standardized incidence ratio of 8.95. This risk increased with age, with an incidence ratio of 13.2 in women over 50 years of age (19). This study emphasizes the difficulties associated with a diagnosis of malignancies arising from endometriosis.

\section{Endometrioid adenocarcinoma of the ovary}

Compared with the general population, women with endometriosis have a higher risk of developing epithelial ovarian cancer. The association between endometriosis and endometrioid and clear cell carcinoma of the ovary has been frequently described in the medical literature and is much stronger than that of serous and mucinous tumors (Fig. 2) (9). Endometriosis-associated ovarian cancer generally affects women who are younger, diagnosed in earlier stages, have lower grade lesions and a better prognosis than those affected by ovarian cancer without endometriosis (20). The majority of cases of endometrioid adenocarcinoma typically arise from endometriosis. It is common to find positivity for estrogen and progesterone receptors, suggesting that estrogen stimulation is somehow involved in tumor pathogenesis (9). Estrogen receptor expression is significantly more common in endometrioid than serous adenocarcinomas and clear cell carcinoma of the ovary. Tamoxifen, as a result of its estrogenic effects, may cause malignant changes in endometriosis (21). Martin also reported that there may be an increased risk of developing endometrioid adenocarcinoma in hormone replacement management in patients with endometriosis (22).

Endometrioid adenocarcinoma of the ovary exhibits activation of Wnt signaling and somatic mutations of CTNNB1 [encoding $\beta$-catenin [cadherin-associated protein)], PTEN (phosphatase and tensin homolog) and PIK3CA 


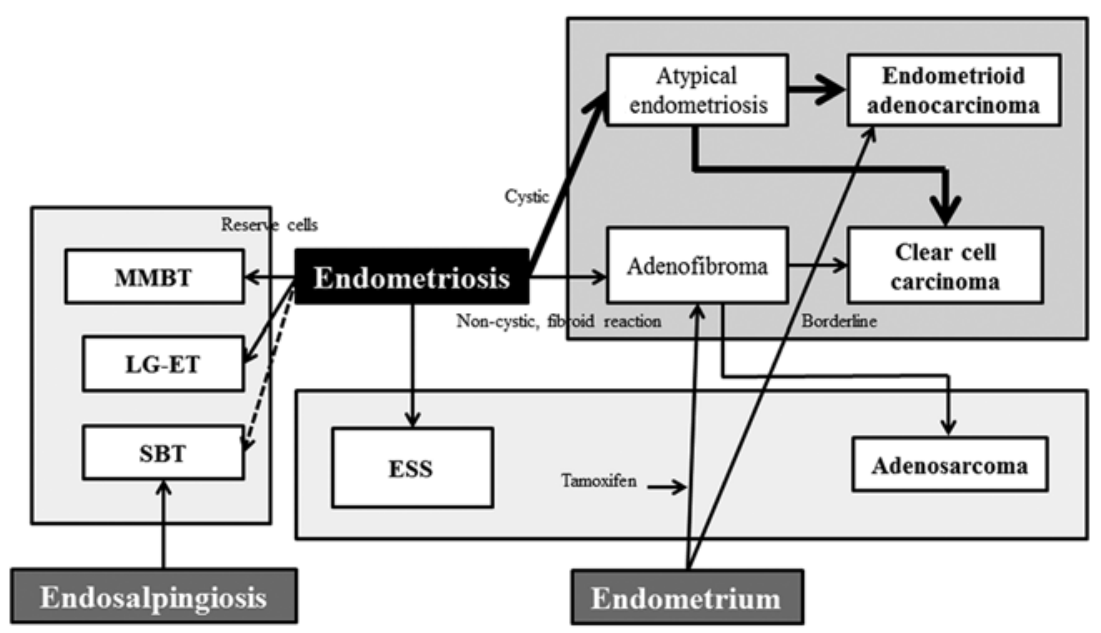

Figure 2. Endometriosis contributes to tumorigenesis via three major pathways. The malignant processes that are associated with endometriosis can be classified into three groups: i) epithelial ovarian cancers (endometrioid adenocarcinoma and clear cell carcinoma), ii) other Müllerian-type tumors (MMBT, SBT and LG-ET), as well as iii) sarcomas (adenosarcoma and ESS) in the female pelvic cavity.

(phosphoinositide-3-kinase, catalytic, $\alpha$ polypeptide) (23). Wnt/ $\beta$-catenin pathway defects are key contributing factors in the cancer phenotype. Recently, Wiegand et al (12) and Jones et al (24) published new data suggesting AT-rich interactive domain-containing protein 1A (ARID1A) as a tumor-suppressor gene disrupted in ovarian clear cell and endometrioid adenocarcinomas. Other authors also showed that the ARID1A complex interacts with p53 (25). Therefore, ARID1A is a tumor suppressor gene that collaborates with p53 to regulate tumor growth and the cell cycle.

\section{Clear cell carcinoma of the ovary}

The clear cell carcinoma evolution from ovarian endometriosis is similar to the process that occurs in endometrioid adenocarcinoma, a type I tumor (26). In their study, Mandai et al have shown that a stressful microenvironment within the endometrioma may lead to cancer development by inducing unique gene expressions, the so-called 'Clear Cell Carcinoma Signature' (27). Several investigators have hypothesized that collection of blood inside endometriomas would lead to iron-induced oxidative stress on endometriotic cells $(28,29)$. This would explain the reason for endometriosis-associated cancers developing more frequently in the ovarian than the extragonadal sites.

Two types of clear cell carcinomas, non-cystic (adenofibroma) and cystic (endometrioma), appear to be derived from endometriosis (Fig. 2) (26). The former involves the step-wise progression sequence of adenofibromas, atypical proliferative (borderline) tumors, and clear cell carcinoma (26). This adenofibroma-clear cell carcinoma sequence develops from non-cystic endometriosis and is associated with adenofibromatous components (26). The latter arises from ovarian endometrioma and is not associated with an adenofibromatous background (26). The two pathways can overlap. Both forms of clear cell carcinoma development are closely associated with type I tumors. In certain cases, there were foci of benign and borderline endometrioid adenofibroma in the same ovary (21). A synchronous endometrioid endometrial adenocarcinoma has sometimes been observed in the uterus (21).
Endometriosis-associated clear cell carcinoma has a high percentage of PIK3CA-activating mutations and ARID1A inactivation mutations (30). PIK3CA and ARID1A are early events in carcinogenesis, probably initiating the malignant transformation of endometriosis. In addition, the platelet-derived growth factor (PDGF) pathway was activated in the adenofibroma-clear cell carcinoma sequence (31). This suggests biological differences between clear cell carcinomas that arise in association with adenofibroma vs. endometriosis. Recent genome-wide expression analyses have demonstrated the specific expression of hepatocyte nuclear factor (HNF)-1 $\beta$ in endometriosis and clear cell carcinoma, but not endometrioid adenocarcinoma, suggesting that early differentiation into the clear cell lineage occurs in the endometriosis with HNF-1 $\beta$ overexpression (32). The HNF-1 $\beta$-dependent pathway provides new insights into the regulation of apoptosis, the cell cycle, glycogen synthesis and chemoresistance (32). It has been reported that HNF-1 $\beta$ gene might play a role in carcinogenesis, pathogenesis, and the pathophysiology of clear cell carcinoma of the ovary (32).

\section{Serous borderline tumor}

Atypical endometriosis was rarely found in serous borderline tumor (SBT) $(33,34)$. Patients with peritoneal SBT also had typical endosalpingiosis (35). Endosalpingiosis is a benign condition and describes the ectopic growth of ciliated fallopian tubal-type epithelium (Fig. 2). Although peritoneal SBT is a rare entity, it is of note since this disorder shows close macroscopic similarity to endometriosis and pathologically microscopic similarity to other Müllerian proliferations with malignant potential (36).

Several hypotheses have been proposed regarding the development of SBT. The different models can be traced back to at least two pathological theories: implantation theory and metaplasia/embryonic theory. A popular hypothesis concerning the pathogenesis of this rare condition is that endometrial cells or tubal cells are transported by various routes (transtubal, hematogenous, lymphogenous or by direct apposition) and implanted in the affected organ and subsequently have a 
malignant potential. Another hypothesis is that a secondary Müllerian system exists, thus Müllerian ectopic lesions are the result of metaplastic processes in the target organ or from scattered embryonic rest. Previous studies have demonstrated that the origin of the majority of low-grade serous tumors or SBT is presumed to be ectopic Müllerian epithelium (37). Thus, the epithelium of SBT may derive from the salpinx, endometriosis, or ovarian surface epithelium that is capable of Müllerian metaplasia (37). Endosalpingiosis, endometriosis and endocervicosis constitute the triad of non-neoplastic disorders of the Müllerian system (38). Non-neoplastic glandular proliferation showing spontaneous Müllerian differentiation has been described in numerous sites including the vagina, uterine cervix, urinary bladder, appendix, peritoneum, abdominal wall (inguinal channel, umbilicus) and the lymph nodes $(38,39)$.

Gene expression profiling studies suggest that SBT is genetically distinct from high-grade serous cancers (40). Ethnic and geographical variation may exist in the process of SBT carcinogenesis. Extensive molecular work-up demonstrates an increased frequency of KRAS (v-Ki-ras2 Kirsten rat sarcoma viral oncogene homolog), BRAF (v-raf murine sarcoma viral oncogene homolog B1), or ERBB2 (v-erb-b2 erythroblastic leukemia viral oncogene homolog 2) mutations in a subset of low-grade serous carcinomas, and a lack of TP53 (tumor protein p53) mutations $(40,41)$.

\section{Müllerian mucinous borderline tumor}

The mucinous borderline tumors are of intestinal type or Müllerian (endocervical-like) type. The intestinal-type tumors (intestinal-type mucinous borderline tumor, IMBT) are the most common. Although Müllerian metaplasia is well recognized at different sites within the female genital tract, the ovarian mucinous borderline tumor of Müllerian type (MMBT) and mixed epithelial borderline tumor of Müllerian type (MEBT) are uncommon subtypes of ovarian tumors (42). MMBT is composed of endocervical gland-like mucinous cells and ciliated columnar epithelium reminiscent of the fallopian tube (43). MMBT likely arises from endometriosis, possibly through its precursor $(42,44)$, while IMBT is not associated with endometriosis (Fig. 2).

MMBT occurs with a relatively high frequency in women of Japanese descent (43). Compared with IMBT, the characteristic features of MMBT are as follows: more frequent bilateral occurrence, paucilocular cysts, association with endometriosis, absence of pseudomyxoma but a possible association of peritoneal implants and lymph node metastases, and a lower mortality rate (43). Other malignancies are also associated with MMBT. D'Angelo et al reported a female individual in whom a keratinizing squamous cell carcinoma of the ovary had arisen from MMBT (45).

Hamada et al draw attention to the existence of the reserve cell-like cells (RCLCs) in MMBT (42). The similarity to the cervical reserve cells may indicate their potential role as precursor cells that may subsequently differentiate into various Müllerian cell types (42). Immunohistochemically, all cases were reactive for estrogen receptor and progesterone receptor, with no nuclear expression of $\beta$-catenin (46). There was reactivity for cytokeratin (CK)7, but not CK20 (47). KRAS mutations were detected in exon 1 and codon 12 in $69 \%$ of cases $(46,48)$. No PTEN mutation was identified in any of the nine exons and PTEN immunoreactivity was diffuse in the nuclei of epithelial and underlying stromal cells (46).

\section{Adenosarcoma}

Müllerian adenosarcoma is an uncommon, but not rare, tumor which is characterized by an admixture of benign, but occasionally atypical, epithelial and a malignant, usually lowgrade, stromal component (49). The most common site is the uterine corpus endometrium (50). Adenosarcoma sometimes includes the lower uterine segment and endocervix. There are also reports on adenosarcoma arising from endometriosis (Fig. 2) $(51,52)$. Of note is the association between adenosarcoma and endometriosis, especially in extrauterine forms (9).

Risk factors include pelvic irradiation, hyperestrogenism, such as after prolonged estrogen stimulation or long-term oral contraceptive use, and the use of tamoxifen and tremifen for breast cancer (53). Toremifene may be effective in cases resistant to tamoxifen. Among the tamoxifen-induced uterine malignancies, the most common uterine malignancy is endometrial adenocarcinoma. Chung et al reported a case of an adenosarcoma after toremifene treatment in a breast cancer patient (54). After one year of toremifene treatment, the patient developed adenofibroma. After an additional four years of treatment, this lesion was transformed into adenosarcoma (54).

Adenosarcoma rarely occurs in the ovary, vagina or fallopian tubes, arising from peritoneal surfaces, or outside the female genital tract, for example in the intestine (50). However, a rare case of a clear cell adenocarcinoma and an adenosarcoma coexisting with a heterologous rhabdomyosarcoma in ovarian endometrioma has been reported (55). Adenosarcoma also arises within the myometrium from adenomyosis.

Uterine sarcomas are relatively rare tumors that account for $1-3 \%$ of female genital tract malignancies and between 4-9\% of uterine neoplasms (49). Uterine sarcomas are divided into the following pathological types: carcinosarcomas $(50 \%)$, leiomyosarcomas $(30 \%)$, endometrial stromal sarcomas (10-15\%), and adenosarcomas (5-10\%). The increased incidence rate of uterine sarcomas is due to an increase of carcinosarcomas. These neoplasms may be divided into two major groups: i) sarcomas showing specific genetic alterations (the EWSR1-FLI1 gene fusion in Ewing's sarcoma), and ii) sarcomas showing multiple and variable gene alterations without a specific pattern (leiomyosarcoma and osteosarcoma).

The biological behaviors of adenosarcoma show low malignant potential (56). Some of the tumors currently classified as adenofibromas are, in fact, well-differentiated adenosarcomas (57). The prognosis in endometriosis-associated tumors is far better than in unassociated mixed Müllerian tumors (9). However, Müllerian adenosarcoma with sarcomatous overgrowth is an extremely aggressive variant, even when diagnosed in its early stage (49). The prognosis in typical adenosarcomas without sarcomatous overgrowth is similar to that of adenofibromas associated with favorable outcome (57). Müllerian adenosarcomas with sarcomatous overgrowth showed strong immunoreactivity for Ki-67 and p53 and loss of CD10 and progesterone receptor immunostaining. 


\section{Endometrial stromal sarcoma}

Endometrial stromal tumors are rare uterine neoplasms including benign stromal nodules, low-grade endometrial stromal sarcomas (ESS), and undifferentiated endometrial sarcomas (58). The origin of ESS is explained by at least two hypotheses. One is the malignant transformation of endometriosis (Fig. 2) (59). There have been some reports in which ESS is considered to be associated with endometriosis (59). However, the exact incidence of ESS in endometriosis is not well described. The nodules were composed of foci of adenomyosis and endometriosis (with focal atypical complex hyperplasia) associated with a stromal spindle cell population immunoreactive for estrogen and progesterone receptors (60).

The other theory is a de novo tumor, potentially derived from Müllerian cells (59). A report suggests that ESS is derived from submesothelial pluripotential cells (61). The pluripotential Müllerian epithelium is considered to be widely distributed in the abdominal and pelvic cavities. Therefore, ESS may originate from endometriosis or from metaplasia of the pelvic Müllerian cells (62). Among patients with ESS of ovarian origin, $11 \%$ were reported to have succumbed to disease, compared to $38 \%$ of patients with ESS of extraovarian origin, suggesting that extraovarian origin indicates a poor prognosis (63).

Immunohistochemical features of ESS include reactivity to estrogen receptors, progesterone receptors, aromatase and CD10 $(21,61,64)$. Immunohistochemical characteristics were not consistent with Müllerian adenocarcinoma with sarcomatous overgrowth. In a previous study, a gene fusion on chromosome 7 that includes two zinc-finger genes (JAZF1 and JJAZ1) was identified in ESS (58). This genetic abnormality is specific to ESS (58). Furthermore, loss of heterozygosity of tumor suppressor genes and deregulation of the Wnt signaling pathway have also been found to be involved in ESS tumorigenesis (65). Notably, the Wnt signaling inhibitor might play a role in the development of endometriosis (66).

\section{Discussion}

Malignant transformation of endometriosis has been described in numerous case reports and reviews of the literature. Endometriosis-associated tumors that involve the female pelvic cavity, i.e., the peritoneum, ovary and fallopian tubes are a diverse group of disorders that range in biological behavior from benign to malignant. Approximately $1.0 \%$ of women with endometriosis have lesions that undergo malignant transformation $(19,67)$. Anatomically, lesions are divided into gonadal $(80 \%)$ or extragonadal $(20 \%)$ lesions $(17,67)$.

Endometriosis-associated epithelial ovarian cancers, endometrioid adenocarcinoma and clear cell carcinoma, are the most common tumors. Furthermore, other Müllerian-type borderline tumors [MMBT, SBT and low-grade endometrial tumors (LG-ET)] may be associated with endometriosis. Studies regarding the association of sarcoma (adenosarcoma and ESS) with endometriosis have been conducted. However, the relationship between endometriosis and extraovarian malignancy, although noteworthy, has yet to be investigated.

Knowledge of clinicopathological features of secondary lesions is essential for the identification, differential diagnosis and treatment strategies of endometriosis-associated malignancies. When these tumors arise from endometriosis, they are usually identified at an early stage and are often of low grade, compared with tumors without endometriosis. Furthermore, a better prognosis and lower mortality rate are the characteristic features. In some patients, the diagnosis is relatively obvious since there is clinical, imaging, or pathological evidence of endometriosis. However, on occasion it may be necessary to distinguish this disease from primary pelvic lesions such as malignant mesothelioma, primary epithelial and mesenchymal tumors, or primary ovarian and peritoneal high-grade serous carcinoma, as well as metastatic tumors. Knowledge of histology, pathology and molecular genetics is essential in the differential diagnosis of endometriosis-associated malignancies.

Individual glands of the endometriotic lesions are genetically derived from single precursor cells, suggesting that endometriosis essentially expands monoclonally (68). Endometriotic lesions would harbor somatic genetic changes in chromosomal regions that supposedly contain genes involved in tumorigenesis. A molecular continuum and lineage between the benign precursor and the malignant entity require strong evidence of common mutational events. Endometriosis and cancer may share similar risk factors. These findings would be consistent with the progression model for tumorigenesis from the benign precursor to malignant lesions.

Genome-wide gene expression profiling studies have provided a first step for identifying molecules key to the characteristics of endometriosis-associated neoplasms. Several investigators have also generated mouse models that spontaneously develop carcinoma similar to human ovarian cancer to understand the molecular genetic events underlying carcinogenesis. Multiple genetic alterations play a role in the pathogenesis of endometriosis-associated neoplams. For example, a variety of molecular events, such as p53 alteration, PTEN silencing, KRAS activation mutation, ARID1A inactivation mutation, PIK3CA activation mutation and HNF-1 $\beta$ activation, have been identified in endometriosis-associated epithelial ovarian cancers (27). KRAS, BRAF, or ERBB2 mutations have been reported in SBT $(40,41)$. MMBT also showed KRAS mutations $(46,48)$. Wnt signaling dysregulation has been suggested to play a role in ESS tumorigenesis (65).

Although a number of crucial pathways involved in carcinogenesis have been identified, knowledge of the early steps remains poorly understood. Mandai et al found that microenvironmental factors, including oxidative stress and inflammation, are crucial in endometriosis-associated ovarian carcinogenesis (27). Retrograde menstruation or ovarian hemorrhage carries highly pro-oxidant factors, such as heme and iron, into the peritoneal cavity or ovarian endometrioma (29). A histologically normal ectopic endometrium may bear genetic damage caused by iron-dependent oxidative stress (29). Persistent oxidative stress has been associated with carcinogenesis $(69,70)$. Iron overload is considered as one such condition that causes oxidative stress via the Fenton reaction, thus promoting the generation of the undesirable free radical molecule, ${ }^{\circ} \mathrm{OH}$ (69). Abnormal iron uptake leads to lipids, proteins and DNA damage derived from free radical toxicity. In general, hemochromatosis, chronic viral hepatitis, inflammatory bowel diseases such as Crohn's disease and ulcerative colitis or asbestosis induce iron overload, which may lead to 
hepatocellular carcinoma, colon cancer or mesothelioma in humans (69). Several investigators have demonstrated that iron-induced oxidative stress is also able to induce specific gene modifications in endometriosis-associated ovarian cancer $(27,28,71-74)$. Undefined molecular events occurring in endometriosis that has undergone Müllerian metaplasia may initiate neoplastic change towards not only carcinoma, but also sarcoma. Although the molecular changes in other Mülleriantype tumors and sarcomas remain largely unknown, DNA damage caused by oxidative stress may be a critical factor in the early event of the malignant transformation process of endometriosis.

In conclusion, the malignant processes that are associated with endometriosis may be classified into: i) epithelial ovarian cancers (endometrioid adenocarcinoma and clear cell carcinoma), ii) other Müllerian-type tumors (MMBT, SBT and LG-ET), as well as iii) sarcomas (adenosarcoma and ESS) in the female pelvic cavity (Fig. 2). The molecular pathology of endometriosis-associated tumorigenesis is heterogeneous and may involve various putative precursor lesions and multiple pathways of development, possibly via genetic alteration by oxidative stress.

\section{Acknowledgements}

This study was supported by a grant [Grant-in-aid for Scientific Research (H. Kobayashi)] from the Ministry of Education, Science, and Culture of Japan.

\section{References}

1. Rogers PA, D'Hooghe TM, Fazleabas A, et al: Priorities for endometriosis research: recommendations from an international consensus workshop. Reprod Sci 16: 335-346, 2009.

2. Gemmill JA, Stratton P, Cleary SD, Ballweg ML and Sinaii N: Cancers, infections, and endocrine diseases in women with endometriosis. Fertil Steril 94: 1627-1631, 2010.

3. Al-Jefout M, Tokushige N, Hey-Cunningham AJ, et al: Microanatomy and function of the eutopic endometrium in endometriosis. Expert Rev Obstet Gynecol 4: 61-79, 2009.

4. Brinton LA, Gridley G, Persson I, Baron J and Bergqvist A: Cancer risk after a hospital discharge diagnosis of endometriosis Am J Obstet Gynecol 176: 572-579, 1997.

5. Brinton LA, Lamb EJ, Moghissi KS, et al: Ovarian cancer risk associated with varying causes of infertility. Fertil Steril 82: 405-414, 2004

6. Kokcu A: Relationship between endometriosis and cancer from current perspective. Arch Gynecol Obstet 284: 1473-1479, 2011.

7. Nezhat F, Datta MS, Hanson V, Pejovic T, Nezhat C and Nezhat C: The relationship of endometriosis and ovarian malignancy: a review. Fertil Steril 90: 1559-15570, 2008.

8. Grammatikakis I, Zervoudis S, Evangelinakis $\mathrm{N}$ and Tziortzioti V: Endometrium and ovarian cancer synchronous to endometriosis - a retrospective study of our experience of 7 years. J Med Life 3: 76-79, 2010.

9. Stern RC, Dash R, Bentley RC, Snyder MJ, Haney AF and Robboy SJ: Malignancy in endometriosis: frequency and comparison of ovarian and extraovarian types. Int $\mathbf{J}$ Gynecol Pathol 20: 133-139, 2001.

10. Mabrouk M, Vicenzi C, Ferrini G, et al: Mixed adenocarcinoma of the rectovaginal septum associated with endometriosis and endometrial carcinoma: a case report. Case Rep Oncol 4: 149-154, 2011

11. Veiga-Castelli LC, Silva JC, Meola J, et al: Genomic alterations detected by comparative genomic hybridization in ovarian endometriomas. Braz J Med Biol Res 43: 799-805, 2010.

12. Wiegand KC, Shah SP, Al-Agha OM, et al: ARID1A mutations in endometriosis-associated ovarian carcinomas. N Engl J Med 363: 1532-1543, 2010.
13. Yamamoto $\mathrm{S}$, Tsuda $\mathrm{H}$, Takano $\mathrm{M}$, Iwaya $\mathrm{K}$, Tamai $\mathrm{S}$ and Matsubara O: PIK3CA mutation is an early event in the development of endometriosis-associated ovarian clear cell adenocarcinoma. J Pathol 225: 189-194, 2011.

14. Scott RB: Malignant changes in endometriosis. Obstet Gynecol 2: 283-289, 1953.

15. Scully RE, Richardson GS and Barlow JF: The development of malignancy in endometriosis. Clin Obstet Gynecol 9: 384-411, 1966.

16. Omranipour R and Najafi M: Papillary serous carcinoma arising in abdominal wall endometriosis treated with neoadjuvant chemotherapy and surgery. Fertil Steril 93: 1347.e17-e18, 2010.

17. Heaps JM, Nieberg RK and Berek JS: Malignant neoplasms arising in endometriosis. Obstet Gynecol 75: 1023-1028, 1990.

18. Sayasneh A, Tsivos D and Crawford R: Endometriosis and ovarian cancer: a systematic review. ISRN Obstet Gynecol 2011: 140310, 2011.

19. Kobayashi H, Sumimoto K, Moniwa N, et al: Risk of developing ovarian cancer among women with ovarian endometrioma: a cohort study in Shizuoka, Japan. Int J Gynecol Cancer 17: 37-43, 2007.

20. Takeuchi M, Matsuzaki K, Uehara H and Nishitani H: Malignant transformation of pelvic endometriosis: MR imaging findings and pathologic correlation. Radiographics 26: 407-417, 2006.

21. McCluggage WG, Bryson C, Lamki H and Boyle DD: Benign, borderline, and malignant endometrioid neoplasia arising in endometriosis in association with tamoxifen therapy. Int J Gynecol Pathol 19: 276-279, 2000

22. Martin DC: Cancer and endometriosis: do we need to be concerned? Semin Reprod Endocrinol 15: 319-324, 1997.

23. Cho KR and Shih IeM: Ovarian cancer. Annu Rev Pathol 4: 287-313, 2009.

24. Jones S, Wang TL, Shih IeM, et al: Frequent mutations of chromatin remodeling gene ARID1A in ovarian clear cell carcinoma. Science 330: 228-231, 2010.

25. Guan B, Wang TL and Shih IM: ARID1A, a factor that promotes formation of SWI/SNF-mediated chromatin remodeling, is a tumor suppressor in gynecologic cancers. Cancer Res 71: 6718-6727, 2011.

26. Zhao C, Wu LS and Barner R: Pathogenesis of ovarian clear cell adenofibroma, atypical proliferative (borderline) tumor, and carcinoma: clinicopathologic features of tumors with endometriosis or adenofibromatous components support two related pathways of tumor development. J Cancer 2: 94-106, 2011.

27. Mandai M, Matsumura N, Baba T, Yamaguchi K, Hamanishi J and Konishi I: Ovarian clear cell carcinoma as a stress-responsive cancer: influence of the microenvironment on the carcinogenesis and cancer phenotype. Cancer Lett 310: 129-133, 2011.

28. Yamaguchi K, Mandai M, Toyokuni S, et al: Contents of endometriotic cysts, especially the high concentration of free iron, are a possible cause of carcinogenesis in the cysts through the ironinduced persistent oxidative stress. Clin Cancer Res 4: 32-40, 2008.

29. Kobayashi H, Kajiwara H, Kanayama S, et al: Molecular pathogenesis of endometriosis-associated clear cell carcinoma of the ovary (Review). Oncol Rep 22: 233-240, 2009.

30. Kuo KT, Mao TL, Jones S, et al: Frequent activating mutations of PIK3CA in ovarian clear cell carcinoma. Am J Pathol 174: 1597-1601, 2009

31. Yamamoto S, Tsuda H, Takano M, et al: Expression of plateletderived growth factors and their receptors in ovarian clear-cell carcinoma and its putative precursors. Mod Pathol 21: 115-124, 2008.

32. Kobayashi H, Yamada Y, Kanayama S, et al: The role of hepatocyte nuclear factor-1 $\beta$ in the pathogenesis of clear cell carcinoma of the ovary. Int J Gynecol Cancer 19: 471-479, 2009.

33. Fukunaga M, Nomura K, Ishikawa E and Ushigome S: Ovarian atypical endometriosis: its close association with malignant epithelial tumours. Histopathology 30: 249-255, 1997.

34. Srinivasan R, Gupta N and Gupta I: Recurrent endocervicallike mucinous borderline tumor (ELMBT) arising in a case of long-standing endometriosis - a case report. Indian J Pathol Microbiol 49: 277-278, 2006.

35. Biscotti CV and Hart WR: Peritoneal serous micropapillomatosis of low malignant potential (serous borderline tumors of the peritoneum). A clinicopathologic study of 17 cases. Am J Surg Pathol 16: 467-745, 1992.

36. Tinelli A, Malvasi A and Pellegrino M: An incidental peritoneal serous borderline tumor during laparoscopy for endometriosis. Eur J Gynaecol Oncol 30: 579-582, 2009. 
37. Carlson J, Roh MH, Chang MC and Crum CP: Recent advances in the understanding of the pathogenesis of serous carcinoma: the concept of low- and high-grade disease and the role of the fallopian tube. Diagn Histopathol (Oxford) 14: 352-365, 2008.

38. McCoubrey A, Houghton O, McCallion K and McCluggage WG: Serous adenocarcinoma of the sigmoid mesentery arising in cystic endosalpingiosis. J Clin Pathol 58: 1221-1223, 2005.

39. Cil AP, Atasoy P and Kara SA: Myometrial involvement of tumor-like cystic endosalpingiosis: a rare entity. Ultrasound Obstet Gynecol 32: 106-110, 2008.

40. Kurman RJ and Shih IeM: The origin and pathogenesis of epithelial ovarian cancer: a proposed unifying theory. Am J Surg Pathol 34: 433-443, 2010

41. Mayr D, Hirschmann A, Löhrs U and Diebold J: KRAS and BRAF mutations in ovarian tumors: a comprehensive study of invasive carcinomas, borderline tumors and extraovarian implants. Gynecol Oncol 103: 883-887, 2006.

42. Hamada T, Kiyokawa T, Nomura K and Hano H: Immunohistochemical analysis of reserve cell-like cells of ovarian müllerian mucinous/mixed epithelial borderline tumor. Int J Gynecol Pathol 27: 199-206, 2008

43. Moriya T, Mikami Y, Sakamoto K, et al: Endocervical-like mucinous borderline tumors of the ovary: clinicopathological features and electron microscopic findings. Med Electron Microsc 36: 240-246, 2003.

44. Lee KR and Nucci MR: Ovarian mucinous and mixed epithelial carcinomas of mullerian (endocervical-like) type: a clinicopathologic analysis of four cases of an uncommon variant associated with endometriosis. Int J Gynecol Pathol 22: 42-51, 2003.

45. D'Angelo E, Dadmanesh F, Pecorelli S and Prat J: Squamous cell carcinoma of the ovary arising from a mucinous cystic tumor of endocervical (müllerian) type. Int J Gynecol Pathol 29: 529-532, 2010.

46. Kim KR, Choi J, Hwang JE, et al: Endocervical-like (Müllerian) mucinous borderline tumours of the ovary are frequently associated with the KRAS mutation. Histopathology 57: 587-596, 2010.

47. Shin JH, Bae JH, Lee A, et al: CK7, CK20, CDX2 and MUC2 Immunohistochemical staining used to distinguish metastatic colorectal carcinoma involving ovary from primary ovarian mucinous adenocarcinoma. Jpn J Clin Oncol 40: 208-213, 2010.

48. Auner V, Kriegshäuser G, Tong D, et al: KRAS mutation analysis in ovarian samples using a high sensitivity biochip assay. BMC Cancer 9: 111, 2009.

49. Patrelli TS, Gizzo S, Di Gangi S, Guidi G, Rondinelli M and Nardelli GB: Cervical Mullerian adenosarcoma with heterologous sarcomatous overgrowth: a fourth case and review of literature. BMC Cancer. 11: 236, 2011.

50. McCluggage WG: Mullerian adenosarcoma of the female genital tract. Adv Anat Pathol 17: 122-129, 2010.

51. Han X, Leng J, Guo L, Xiang Y and Lang J: Vaginal adenosarcoma arising from refractory endometriosis: a case report. Aust N Z J Obstet Gynaecol 50: 574-576, 2010.

52. Patrelli TS, Silini EM, Gizzo S, et al: Extragenital Müllerian adenosarcoma with pouch of Douglas location. BMC Cancer 11: 171, 2011.

53. Singh R, Shameema S, Vijaya K and Kumar P: Mullerian adenosarcoma of the uterus with sarcomatous overgrowth. Clin Med Insights Case Rep 3: 27-30, 2010.

54. Chung YW, Bae HS, Han SI, Song JY, Kim IS and Kang JS Endometrial mullerian adenosarcoma after toremifene treatment in breast cancer patients: a case report. J Gynecol Oncol 21: 269-272, 2010.

55. Yasuoka H, Tsujimoto M, Fujita S, et al: Coexistence of a clear cell adenocarcinoma and an adenosarcoma with a heterologous rhabdomyosarcoma in an endometriotic cyst of the ovary: a case study. Int J Gynecol Pathol 28: 362-366, 2009.
56. Clement PB and Scully RE: Mullerian adenosarcoma of the uterus. A clinicopathologic analysis of ten cases of a distinctive type of mullerian mixed tumor. Cancer 34: 1138-1149, 1974.

57. Gallardo A and Prat J: Mullerian adenosarcoma: a clinicopathologic and immunohistochemical study of 55 cases challenging the existence of adenofibroma. Am J Surg Pathol 33: 278-288, 2009.

58. Hrzenjak A, Moinfar F, Tavassoli FA, et al: JAZF1/JJAZ1 gene fusion in endometrial stromal sarcomas: molecular analysis by reverse transcriptase-polymerase chain reaction optimized for paraffin-embedded tissue. J Mol Diagn 7: 388-395, 2005.

59. Jung SI, Shin SS, Choi C, Hwang EC, Kim SO and Kang TW: Endometrial stromal sarcoma presenting as prevesical mass mimicking urachal tumor. J Korean Med Sci 24: 529-531, 2009.

60. Froio E, Piana S, Cavazza A, Valli R, Abrate M and Gardini G: Multifocal PEComa (PEComatosis) of the female genital tract associated with endometriosis, diffuse adenomyosis, and endometrial atypical hyperplasia. Int J Surg Pathol 16: 443-446, 2008

61. Kim JY, Hong SY, Sung HJ, Oh HK and Koh SB: A case of multiple metastatic low-grade endometrial stromal sarcoma arising from an ovarian endometriotic lesion. J Gynecol Oncol 20: 122-125, 2009.

62. Khan AW, Craig M, Jarmulowicz M and Davidson BR: Liver tumours due to endometriosis and endometrial stromal sarcoma. HPB (Oxford) 4: 43-45, 2002.

63. Baiocchi G, Kavanagh JJ and Wharton JT: Endometrioid stromal sarcomas arising from ovarian and extraovarian endometriosis: report of two cases and review of the literature. Gynecol Oncol 36: 147-151, 1990.

64. Roşca E, Venter A, Muţiu G, Drăgan A, Coroi M and Roşca DM: Endometrial stromal sarcoma developed on outer endometriosis foci. Rom J Morphol Embryol 52: 489-492, 2011.

65. Chiang S and Oliva E: Cytogenetic and molecular aberrations in endometrial stromal tumors. Hum Pathol 42: 609-617, 2011.

66. Cheng CW, Smith SK and Charnock-Jones DS: Transcript profile and localization of Wnt signaling-related molecules in human endometrium. Fertil Steril 90: 201-204, 2008.

67. Irvin W, Pelkey T, Rice L and Andersen W: Endometrial stromal sarcoma of the vulva arising in extraovarian endometriosis: a case report and literature review. Gynecol Oncol 71: 313-316, 1998.

68. Viganó P, Somigliana E, Chiodo I, Abbiati A and Vercellini P: Molecular mechanisms and biological plausibility underlying the malignant transformation of endometriosis: a critical analysis. Hum Reprod Update 12: 77-89, 2006.

69. Toyokuni S: Mysterious link between iron overload and CDKN2A/2B. J Clin Biochem Nutr 48: 46-49, 2011.

70. Toyokuni S: Molecular mechanisms of oxidative stressinduced carcinogenesis: from epidemiology to oxygenomics. IUBMB Life 60: 441-447, 2008.

71. Kajihara H, Yamada Y, Kanayama S, et al: Clear cell carcinoma of the ovary: Potential pathogenic mechanisms. Oncol Rep 23: 1193-1203, 2010

72. Yamada Y, Shigetomi H, Onogi A, et al: Redox-active ironinduced oxidative stress in the pathogenesis of clear cell carcinoma of the ovary. Int J Gynecol Cancer 21: 1200-1207, 2011.

73. Mandai M, Yamaguchi K, Matsumura N, Baba T and Konishi I: Ovarian cancer in endometriosis: molecular biology, pathology, and clinical management. Int J Clin Oncol 14: 383-391, 2009

74. Yamaguchi K, Mandai M, Oura T, et al: Identification of an ovarian clear cell carcinoma gene signature that reflects inherent disease biology and the carcinogenic processes. Oncogene 29: 1741-1752, 2010. 